\title{
A NOTE ON THE COINCIDENCE OF SOME RANDOM FUNCTIONS*
}

By GEORGE H. WEISS (Ballistic Research Laboratories, Aberdeen Proving Ground, Md.)

Abstract. This paper deals with a system capable of randomly attaining two states "on" and "off". For convenience this system is pictured as a pulse train. The question is, what is the expected amount of time that $n$ of such systems are in the same state simultaneously. The answer is shown to depend on the probability of a system being in a given state at a specified time. A formal solution for the Laplace transform of this probability is given.

In this paper we shall consider a mathematical problem arising out of the following situation: Suppose two or more new machines (relays, vacuum tubes, electric bulbs) are installed at time $t=0$, and are operated continuously thereafter. Whenever any of the machines break down it is replaced by a new one. Both the operating time of the machine and the replacement time are described by probability densities which are defined below. We wish to find the expected fraction of time during which all of the machines are being simultaneously replaced.

A somewhat similar problem was considered ${ }^{1,2}$ in connection with the coincidence of periodic pulse trains coming from radar systems.

Let us therefore consider a system which is capable of existing in two states, say "on" and "off". For convenience we may picture it as a train of pulses, as shown in Fig. 1. As seen from the figure, the length of a single "on" pulse will be denoted by $l_{k}$ (the subscript $k$ referring to the $k$ th of $n$ pulse trains) and the length of a single "off" pulse will be denoted by $m_{k}$. We shall assume that the probability that an "on" pulse from the $k$ th pulse train ends in the interval $\left(l_{k}, l_{k}+d l_{k}\right)$ is given by $\alpha_{k}\left(l_{k}\right) d l_{k}$. Similarly

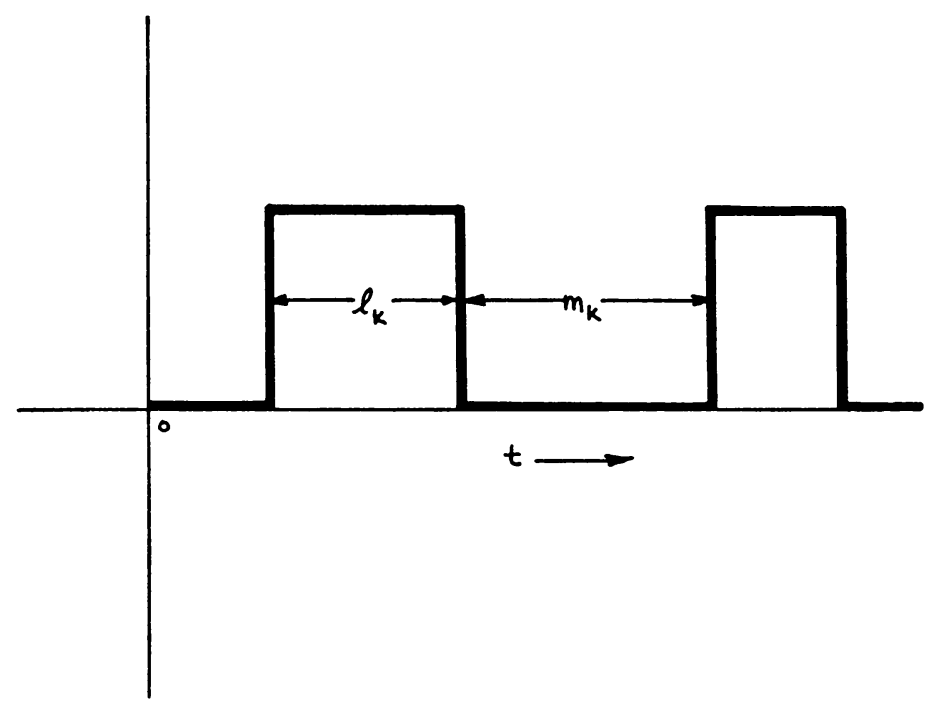

Fig. 1. Nomenclature for the $k$ th pulse train.

*Received April 5, 1955.

${ }^{1}$ K. S. Miller and R. J. Schwartz, J. Appl. Phys. 24, 1032-1036 (1953).

${ }^{2}$ H. D. Friedman, J. Appl. Phys. 25, 1001-1005 (1954). 
the probability just an "off" pulse end in the interval $\left(m_{k}, m_{k}+d m_{k}\right)$ is $\beta_{k}\left(m_{k}\right) d m_{k}$. Then, by definition we have

$$
\begin{gathered}
\int_{0}^{\infty} \alpha_{k}(l) d l=1 \\
\int_{0}^{\infty} \beta_{k}(m) d m=1 .
\end{gathered}
$$

We may now ask, what is the expected fraction of time during which $n$ "on" pulses, one from each of $n$ trains coincide? For the answer to this question we will assume it possible to find the probability $\eta_{k}(t)$ that a pulse from the $k$ th train is on at time $t$. Then, in the time interval $(0, T)$, the expected fraction of time during which all $n$ pulses are on is

$$
\frac{1}{T} \int_{0}^{T}\left\{\prod_{k=1}^{n} \eta_{k}(t)\right\} d t .
$$

Thus, the solution of the problem depends only on a knowledge of the $\eta_{k}(t)$. The remainder of this paper gives a derivation for $\eta_{k}(t)$ in terms of the fundamental distributions $\alpha_{k}\left(l_{k}\right)$ and $\beta_{k}\left(m_{k}\right)$.

In order to proceed with a solution we introduce the auxiliary probability densities $\omega_{k}(t)$, defined by

$$
\lim _{d t \rightarrow 0} \omega_{k}(t) d t=\lim _{d t \rightarrow 0} \operatorname{Prob}\{\text { pulse ends in time interval }(t, t+d t)\} .
$$

We shall also use the probability, $A_{k}(l)$, that an "on" pulse from the $k$ th train is greater than $l$ in duration. Hence

$$
A_{k}(l)=\int_{l}^{\infty} \alpha_{k}(x) d x
$$

and $A_{k}(0)=1$, by $(1)$.

An expression for $\eta(t)$ (we omit the subscript $k$ in the following calculations) may be derived by reasoning that if a pulse is on at time $t$, then it is either the first pulse or a subsequent one. The probability $\eta(t)$ is therefore

$$
\eta(t)=\int_{0}^{t} \beta(\tau) A(t-\tau) d \tau+\int_{0}^{t} \omega\left(\tau_{1}\right) d \tau_{1} \int_{\tau_{1}}^{t} \beta\left(\tau-\tau_{1}\right) A(t-\tau) d \tau
$$

or

$$
\eta(t)=\lambda(t)+\int_{0}^{t} \omega(\tau) \lambda(t-\tau) d \tau
$$

with

$$
\lambda(t)=\int_{0}^{t} \beta(\tau) A(t-\tau) d \tau .
$$

An expression for $\omega(t)$ is found in similar fashion. If an "on" pulse ends in the interval $(t, t+d t)$, it must be the first of such endings or a later one, hence

$$
\omega(t)=\phi(t)+\int_{0}^{t} \omega(\tau) \phi(t-\tau) d \tau,
$$


where

$$
\phi(t)=\int_{0}^{t} \beta(\tau) \alpha(t-\tau) d \tau
$$

Since both (5) and (8) are equations of the convolution type, we may simplify results by using Laplace transforms. The notation will be used

$$
L\{G(t)\}=\int_{0}^{\infty} e^{-\bullet t} G(t) d t=G^{*}(s)
$$

The transform $L\{A(l)\}$ is given by

$$
L\{A(l)\}=\frac{1-\alpha^{*}(s)}{s}
$$

so that, from (5) and (8)

$$
\eta^{*}(s)=\frac{\beta^{*}(s)}{s}\left[\frac{1-\alpha^{*}(s)}{1-\alpha^{*}(s) \beta^{*}(s)}\right]
$$

and

$$
\eta(t)=\frac{1}{2 \pi i} \int_{c-i \infty}^{c+i \infty} \frac{e^{\theta t}}{s} \beta^{*}(s)\left[\frac{1-\alpha^{*}(s)}{1-\alpha^{*}(s) \beta^{*}(s)}\right] d s
$$

which can then be substituted in (2) to give the coincidence fraction.

An example of the use of the preceding formulae is given by a computation of the expected coincidence time fraction of two pulse trains, both described by

$$
\begin{aligned}
\alpha(l) & =a e^{-a l}, \\
\beta(m) & =b e^{-b m},
\end{aligned}
$$

where $a$ and $b$ are constants. Then $\alpha^{*}(s)=a /(s+a)$ and $\beta^{*}(s)=b /(s+b)$. With these transforms we have

$$
\eta^{*}(s)=\frac{b}{s(s+a+b)}
$$

so that

$$
\eta(t)=\frac{b}{a+b}\{1-\exp [-(a+b) t]\}
$$

The probable fraction of time spent in coincidence is

$\frac{1}{T} \int_{0}^{T} \eta^{2}(t) d t$

$$
=\frac{b^{2}}{(a+b)^{2}}\left\{1-\frac{2[1-\exp (-(a+b) T)]}{(a+b) T}+\frac{[1-\exp (-2(a+b) T)]}{2(a+b) T}\right\} .
$$

As $T \rightarrow \infty$, this approaches $b^{2} /(a+b)^{2}$.

For many applications it is unnecessary to give a detailed evaluation of the integral 
(2) but rather an asymptotic value as $T \rightarrow \infty$. In the case that each $\eta_{k}(t)$ approaches a constant $C_{k}$ for $t \rightarrow \infty$, it follows that

$$
\lim _{T \rightarrow \infty} \frac{1}{T} \int_{0}^{r}\left\{\prod_{k=1}^{n} \eta_{k}(t)\right\} d t=\prod_{k=1}^{n} C_{k} .
$$

Under certain conditions on the $\alpha_{k}(l)$ and $\beta_{k}(m)$ it is possible to show that lim $\rightarrow \rightarrow \infty$ $\eta_{k}(t) \rightarrow C_{k}$, where, in fact, $C_{k}$ is a function of the first moments of $\alpha_{k}(l)$ and $\beta_{k}(m)$. We shall give sufficient conditions for $\eta_{k}(t)$ to approach a constant as $t \rightarrow \infty$ and then derive the value of $C_{k}$. It will be shown in the following remarks that in order for $\eta_{k}(t)$ to be asymptotic to a constant, it is sufficient that $\omega_{k}(t)$ be asymptotic to a constant. Sufficient conditions for $\omega_{k}(t)$ to be asymptotic to a constant will then be quoted from the work of Feller ${ }^{3}$.

At the outset let us make the following assumptions:

(a) $\alpha_{k}(t), \beta_{k}(t) \rightarrow 0$ as $t \rightarrow \infty$.

(b) The first moments, written $\left\langle l_{k}\right\rangle,\left\langle m_{k}\right\rangle$, and defined by

$$
\begin{aligned}
& \left\langle l_{k}\right\rangle=\int_{0}^{\infty} l \alpha_{k}(l) d l \\
& \left\langle m_{k}\right\rangle=\int_{0}^{\infty} m \beta_{k}(m) d m
\end{aligned}
$$

exist.

(c) $\lim _{t \rightarrow \infty} \lambda_{k}(t)=0$.

From assumption (a) we derive

Leman 1: The function $\phi(t)$, defined in (9), approaches zero as $t \rightarrow \infty$. This lemma is obvious on the consideration that $\phi(t)$ is the probability density for the time $l+m$. With this lemma we prove

Theorem 1: If $\omega(t) \rightarrow B$ as $t \rightarrow \infty$, then $\eta(t) \rightarrow C$ as $t \rightarrow \infty$.

Proof: Write $\omega(t)=B+\Omega(t)$, where, by hypothesis

$$
|\Omega(t)|<\epsilon, \quad \epsilon>0
$$

for $t>N(\epsilon)$. The time $N(\epsilon)$ is also chosen so that

$$
\lambda(t)<\epsilon, \quad t>N(\epsilon)
$$

which is possible because of (c). If $\omega(t)$ satisfies (8), then, with $\alpha(l)$ and $\beta(m)$ chosen to satisfy (b) $\omega(t)$ is a non-negative bounded function ${ }^{4}$, hence $\omega(t)$ is bounded.

From (5) we have

$$
\begin{aligned}
\lim _{t \rightarrow \infty} \eta(t) & =\lim _{t \rightarrow \infty} \phi(t)+\lim _{t \rightarrow \infty} \int_{0}^{t} \omega(\tau) \lambda(t-\tau) d \tau \\
& =\lim _{t \rightarrow \infty}\left\{B \int_{0}^{t} \lambda(\tau) d \tau+\int_{0}^{t} \Omega(\tau) \lambda(t-\tau) d \tau\right\} .
\end{aligned}
$$

'Feller, W., On the integral equation of renewal theory, Annals Math. Statistics 12, 243-267 (1941).

'This is the Theorem 2 given in the last reference. 
The first integral in (22) can be handled by an integration by parts

$$
\lim _{t \rightarrow \infty} \int_{0}^{t} \lambda(\tau) d \tau=\lim _{t \rightarrow \infty}\left\{\lambda(t)+\int_{0}^{t} \tau \alpha(\tau) d \tau\right\},
$$

where the first term on the right hand side is eliminated by the use of (c). Finally

$$
\begin{aligned}
\left|\int_{0}^{t} \Omega(\tau) \lambda(t-\tau) d \tau\right| & \leq\left|\int_{0}^{N} \Omega(\tau) \lambda(t-\tau) d \tau\right|+\left|\int_{N}^{t} \Omega(\tau) \lambda(t-\tau) d \tau\right| \\
& \leq \epsilon\left|\int_{0}^{N} \Omega(\tau) d \tau\right|+\epsilon\left|\int_{N}^{t} \lambda(t-\tau) d \tau\right| \\
& <\epsilon(M+\langle l\rangle),
\end{aligned}
$$

where $M$ is a constant independent of $\epsilon$, so that $\lim _{t \rightarrow \infty} \eta(t)=B\langle l\rangle$.

Sufficient conditions for $\omega(t)$ to be asymptotically constant have been cited by Feller $^{3}$. Based on Tauberian theorems for Laplace transforms, these conditions are
a1) $\int_{0}^{\infty} \phi(t) d t=1$,
a2) $\int_{0}^{\infty} t \phi(t) d t<\infty$,
a3) $\lim _{t \rightarrow \infty} \int_{t}^{\infty} \phi(t) d t=0$.

The conditions (a1), (a2), and (a3) will be satisfied if assumptions (a), (b), and (c) are satisfied by $\alpha_{k}(l)$ and $\beta_{k}(m)$.

The evaluation of $B_{k}$ makes use of the following Tauberian theorem ${ }^{s}:$ If $\omega_{k}(t) \sim B_{k}$ as $t \rightarrow \infty$ then $\omega_{k}^{*}(s) \sim B_{k} / s$ for $s \rightarrow 0+$. The expression for $\omega_{k}(s)$ given in (16) yields the result

$$
\omega_{k}^{*}(s) \sim \frac{1}{s} \cdot \frac{1}{\left\langle l_{k}\right\rangle+\left\langle m_{k}\right\rangle} .
$$

Therefore, if assumptions (a), (b), and (c) are satisfied on $a_{k}(l)$ and $\beta_{k}(m)$ an asymptotic result for the coincidence time fraction, (2) is

$$
\frac{1}{T} \int_{0}^{T}\left\{\prod_{k=1}^{n} \eta_{k}(t)\right\} d t \sim \prod_{k=1}^{n} \frac{\left\langle l_{k}\right\rangle}{\left\langle l_{k}\right\rangle+\left\langle m_{k}\right\rangle} .
$$

In the papers cited in Footnotes 1 and 2, the estimate (27) was not a sufficient one.

Note added in proof: Hypothesis (c) of Eq. (19) is implied by hypothesis (b). This may be seen by the following steps:

$$
t \lambda(t)=-t \int_{t}^{\infty} d \lambda(x) \leq \int_{t}^{\infty} x d \lambda(x) .
$$

However the integral

$$
-\int_{0}^{\infty} x d \lambda(x)
$$

exists and is easily seen to equal $\langle l\rangle$, hence the last integral in (1a) must approach zero as $t$ approaches infinity, which proves the assertion.

'G. Doetsch, Theorie und Anwendung der Laplace Transformation, Dover Publications, N.Y., p. 193. 